\title{
APPLICATION OF LIQUISOLID TECHNOLOGY TO ENHANCE THE DISSOLUTION OF CEFIXIME FROM ITS ORAL CAPSULES
}

\author{
ZAINAB H. MAHDI*, NIDHAL K. MARAIE, ZAHRAA AMER AL-JUBOORI \\ Department of Pharmaceutics, College of Pharmacy, Mustansiriyah University, Baghdad, Iraq
}

Email: zhmpharm@yahoo.com

Received: 07 Jul 2018, Revised and Accepted: 01 Aug 2018

\begin{abstract}
Objective: Oral drug delivery is the most desired route for drug administration for its well-known features. Therefore, many attempts were implemented to improve the poor solubility of many active ingredients in order to enhance their dissolution and absorption via the oral route. From these, the liquisolid system is a very promising technology for enhancing solubility and bioavailability of poorly soluble drugs.

Methods: In this research, oral capsules of cefixime were prepared by liquisolid technique after mixing different concentrations of the drug with propylene glycol (non-volatile solvent), followed by their addition to different proportions of microcrystalline cellulose and aerosil i.e. different carrier: coating (R-value). The liquisolid capsules were evaluated for In vitro disintegration and dissolution in addition to content uniformity and weight variations. Furthermore, solubility studies, scanning electron microscope (SEM) were performed to the optimum formula. Finally, the release profile of the optimum formula was compared with the marketed cefixime capsules.
\end{abstract}

Results: Liquisolid formula (F3) with $70 \%$ cefixime and R-value equals 10 was selected as the optimum formula having higher \% release in 45 min $(99.5 \% \pm 0.53)$ compared to other formulas with faster release rate in the first 20 min than marketed capsules. It had an acceptable disintegration time (25 min \pm 0.76$)$, content uniformity (197.6 \pm 0.92$)$ and weight variation (698.04 \pm 0.16$)$. Results of solubility study, SEM assured enhancement in solubility and dispersibility of the drug.

Conclusion: This research proved that liquisolid system is a promising technology in improving the solubility and dissolution of cefixime from its capsules and hence it may improve its absorption and oral bioavailability.

Keywords: Cefixime trihydrate, Liquisolid, Microcrystalline cellulose

(c) 2018 The Authors. Published by Innovare Academic Sciences Pvt Ltd. This is an open access article under the CC BY license (http://creativecommons.org/licenses/by/4.0/) DOI: http://dx.doi.org/10.22159/ijap.2018v10i5.28359

\section{INTRODUCTION}

Despite of plenty of different routes for administrating a drug, yet oral route solely remains the preferred one, simply because of its convenience, optimum patient compliance as well as low costs of production. However, for a scientist to formulate a drug into, any oral dosage form, a drug must possess sufficient water solubility, therefore, it is quite challenging to formulate any drug in oral dosage form since nearly half of the active substances being identified are either insoluble or poorly soluble in water. The poor dissolution rates of water-insoluble or poorly soluble drugs are significant problem confronting the pharmaceutical industry as it is considered the rate limiting step for absorption and hence for bioavailability.

Different methods are employed to improve the dissolution characteristics of poorly water-soluble drugs like pH adjustment, cosolvents solubilisation, microemulsion, polymeric modification, solid dispersion, drug complexation, micronization, selfemulsification, use of a surfactant as a solubilizing agent, lyophilisation, the pro-drug approach, microencapsulation and inclusion of drug solutions into soft gelatin capsules. Recently, better approaches are available to prepare the liquid oily medications, and drug solutions of water-insoluble solid drugs and the most promising one is the use of the liquisolid system or powder solution technology [1-6].

Liquisolid system is a novel technique developed by Spireas et al., it is a powdered form of liquid drug formulated by dissolving or dispersing the slightly soluble drug in an appropriate non-volatile solvent like polyethylene glycol (PEG) 200 or 400, propylene glycol (PG) or tween 80 , that is absorbed or adsorbed into and onto the carrier like microcrystalline cellulose (MCC) PH 101 or 102 followed by its adsorption onto the surface of suitable coating material like aerosil 200. Hence, the increased surface area of the drug in powder form, enhanced wettability and enhanced dissolution might improve the bioavailability of the drug in the body. This technique was effectively applied to low dose water-insoluble drugs. On the other hand formulation of the high dose was impractical because of the high levels of carrier and coating materials that should be added and in turn will increase the weight of each tablet above $1 \mathrm{~g}$ which is very difficult to swallow. Therefore, to overcome this limitation, some hydrophilic materials can be added to the liquid medication like polyvinylpyrrolidone (PVP), hydroxypropyl methylcellulose or PEG 35000 which produce dry powder formulations containing a liquid with a high concentration of drug using a lower amount of the carrier and coating materials [7-12].

Cefixime is slightly soluble powder with a low bioavailability when given orally (22-54\%), belongs to the third-generation cephalosporin antibacterial and is given at 200 and $400 \mathrm{mg}$ orally in the treatment of susceptible infections, including gonorrhea, otitis media, pharyngitis, lower-respiratory-tract infections such as bronchitis, and urinary tract infections $[13,14]$.

In this study; liquisolid technique was applied to enhance the solubility of cefixime trihydrate using different concentrations of the drug in the non-volatile solvent and different ratios of the carrier and coating materials in an attempt to improve the drug dissolution in comparison to the commercial capsule and hence its oral bioavailability as well as reducing its effective dose.

\section{MATERIALS AND METHODS}

\section{Materials}

Cefixime trihydrate (Lupi Medico, India), MCC PH 102, aerosil 200 (Samara, Iraq), PG (Samara, Iraq), PVP (Samara, Iraq), marketed cefixime $200 \mathrm{mg}$ capsule (Ritemed, Japan). All other chemicals, reagents and solutions used were of analytical grade.

Mathematical modeling for the preparation of the liquisolid system

A mathematical model was employed to accomplish the formulation design of the liquisolid system; such model was described by Spireas 
et al. In this study, PG was used as a liquid vehicle; MCC PH 102 and aerosil 200 were used as the carrier and coating materials, respectively. The concentration of the drug in PG was taken as 70, 75 , and $80 \mathrm{~g} \%$ and the carrier: coat ratios (R) used were 6, 8 and 10. According to new theories, the carrier and coating powder materials can retain only certain amounts of liquid while maintaining acceptable flowability and compressibility.

The weight of carrier powder $(\mathrm{Q})$ in the system was obtained after calculating the weight of liquid medication (W) for each concentration and after obtaining liquid load factors Lf of the formulations for each $\mathrm{R}$ ratio used by the application of the following equations:

$$
\begin{aligned}
& \mathrm{Lf}=\boldsymbol{\Phi}+\Phi(1 / R) \ldots \ldots \ldots \ldots \ldots \ldots \ldots \ldots . . .(1) \\
& \mathrm{Lf}=\mathrm{W} / \mathrm{Q} \text {. }
\end{aligned}
$$

Where, $\Phi$ and $\Phi$ are the flowable liquid retention potential ( $\Phi$ value) of powder excipients which is defined as the maximum weight of liquid that can be retained per unit powder material in order to produce and acceptably flowing liquid/powder admixture.
After that the amount of the coating material (q) in each formula was calculated as follows:

$$
\mathrm{R}=\mathrm{Q} / \mathrm{q}
$$

By using the above mathematical model, liquisolid mixtures were formulated (table 1) first by preparation the liquid medication after dissolving the $200 \mathrm{mg}$ cefixime in $\mathrm{PG}$ at $80{ }^{\circ} \mathrm{C}$ in shaking water bath for $15 \mathrm{~min}$, followed by the addition of $10 \%$ PVP prior to its mixing with the carrier and the coating material using the standard mixing process mentioned by spireas [15-17] by one rotation per second in three mixing steps. The first step involved uniform mixing for about one min to ensure uniform distribution of the liquid medication into the powder, followed by evenly distributing it as a uniform layer on the surface of the mortar and leaving it for about $5 \mathrm{~min}$ to allow the drug solution to be incorporated into and onto the powder. The third step involved the addition of $4 \%$ CCS as a super disintegrant that was mixed for another $30 \mathrm{~s}$ using the same standard mixing process.

The liquisolid mixtures were incorporated after that into capsules

\begin{tabular}{|c|c|c|c|c|c|c|c|}
\hline Formula & $\%$ of drug (w/w) & $\mathbf{R}$ & Lf & W (mg) & Q (mg) & Q (mg) & Total weight (mg) \\
\hline F1 & $70 \%$ & 6 & 0.71 & 285.7 & 200.7 & 33.4 & 601.7 \\
\hline $\mathrm{F} 2$ & $70 \%$ & 8 & 0.57 & 285.7 & 249.3 & 31.1 & 655.2 \\
\hline F3 & $70 \%$ & 10 & 0.49 & 285.7 & 290.9 & 29 & 701 \\
\hline $\mathrm{F} 4$ & $75 \%$ & 6 & 0.71 & 266.6 & 187.3 & 31.2 & 561.6 \\
\hline F5 & $75 \%$ & 8 & 0.57 & 266.6 & 232.6 & 29 & 611.6 \\
\hline F6 & $75 \%$ & 10 & 0.49 & 266.6 & 271.5 & 27.1 & 654.33 \\
\hline F7 & $80 \%$ & 6 & 0.71 & 250 & 175.6 & 29.2 & 526.5 \\
\hline F8 & $80 \%$ & 8 & 0.57 & 250 & 218.1 & 27.2 & 573.3 \\
\hline F9 & $80 \%$ & 10 & 0.49 & 250 & 254.5 & 25.4 & 613.4 \\
\hline
\end{tabular}
by dipping technique to be evaluated.

Table 1: Content of the prepared formulas containing liquisolid cefixime

*Abbreviations: Carrier: coating ratio (R), loading factor $\left(\mathrm{L}_{\mathrm{f}}\right)$, weight of liquid medication $(\mathrm{W})$, weight of carrier (Q), weight of coating material (q).

\section{Evaluation of the liquisolid powder mixture}

\section{Flowability assessment of the liquisolid powder}

The flow properties of liquisolid formulations were studied by an angle of repose, Carr's index and Hausner's ratios. All studies were done in triplicate, where, the angle of repose was calculated by a fixed height cone method, while Carr's index and Hausner's ratios were measured using bulk and tapped densities $[18,19]$.

\section{Solubility study}

The saturated solubility of pure cefixime and optimum liquisolid formula (F3) were studied in distilled water and 7.4 phosphate buffer. An excess amount of each powder was added to each solution and sonicated at room temperature for $1 \mathrm{~h}$, followed by mixing for $24 \mathrm{~h}$ at $25 \pm 0.1{ }^{\circ} \mathrm{C}$. The samples were filtered and evaluated by a UVspectrophotometer at $288 \mathrm{~nm}$ [20].

\section{Scanning electron microscope (SEM)}

The morphological behaviour of the drug alone and the liquisolid system of the optimum formula (F3) was detected by SEM (S-50 model, FEI company-Netherland), where the complete disappearance of the crystals in the liquisolid mixture assures complete solubility of the drug $[21,22]$.

\section{Fourier transform infrared spectroscopy (FT-IR)}

The possible interaction of all excipient along with the drug was assessed for the optimum formula (F3) using FTIR from 4000-400 $\mathrm{cm}^{-1}$ by using the pressed-disk technique [23].

\section{Evaluation of the liquisolid capsules}

\section{Weight variation}

It's done by individually weighing 20 capsules and the average weight is determined. If 2 capsules fall outside the range of $10 \%$, then should take another 20 capsules. So the test requirement is met if 6 capsules fall outside the 10\% range and none of the 6 capsules outside $25 \%$ range.

\section{Content uniformity}

It's performed by and dissolving 10 capsules individually in 7.2 phosphate buffer followed by measuring the amount of cefixime in each capsule by UV-spectrophotometer at $288 \mathrm{~nm}$, the capsules met the requirements if only one falls outside $\pm 15 \%$ but not outside $\pm 25 \%$. If more than two were in that range, then take 20 capsules that is assayed individually, they passed the test, if only 3 of the 30 capsules was outside the range $\pm 15 \%$ but not outside $\pm 25 \%$.

\section{Disintegration test}

Disintegration time (DT) was measured to the prepared liquisolid capsules in $\mathrm{pH} 7.2$ phosphate buffer at $37 \pm 0.5{ }^{\circ} \mathrm{C}$ by placing 6 capsules individually in each tube of the apparatus [24].

\section{Dissolution test}

The test was performed using USP type I apparatus (100 rpm) at $37 \pm 0.5{ }^{\circ} \mathrm{C}$, by placing 3 capsules individually in $1000 \mathrm{ml} \mathrm{pH} 7.2$ phosphate buffer for $45 \mathrm{~min}$. Samples were periodically withdrawn and analyzed spectrophotometrically at $288 \mathrm{~nm}$ and the \% drug release after $45 \mathrm{~min}\left(\% \mathrm{D}_{45} \mathrm{~min}\right)$ was calculated [25].

\section{Statistical analysis}

All the results were statistically analyzed using (ANOVA) single factor to compare and assess the significance of the results, where $(\mathrm{P}<0.05)$ considered to be significant.

\section{RESULTS AND DISCUSSION}

\section{Flowability parameters of the liquisolid powder}

Results of the angle of repose, Carr's index and Hausner's ratio with the corresponding type of flow are illustrated in table 2. According to the USP the results clearly demonstrate that all cefixime liquisolid powder had an acceptable flowability. 
Table 2: Flowability parameters of cefixime liquisolid powder with their corresponding type of flow

\begin{tabular}{lllll}
\hline Formula & Angle of repose & Carr's index & Hausner's ratio \\
\hline F1 & $31.3 \pm 0.32$ & $15.81 \pm 0.96$ & $1.16 \pm 0.25$ & Type of flow \\
F2 & $32.1 \pm 0.65$ & $14.32 \pm 0.66$ & $1.17 \pm 0.21$ & $1.1 \pm 0.94$ \\
F3 & $30.2 \pm 0.95$ & $9.13 \pm 0.93$ & $1.11 \pm 0.83$ & Good \\
F4 & $29.9 \pm 0.76$ & $9.41 \pm 0.9$ & $1.18 \pm 0.53$ & Excellent \\
F5 & $32.5 \pm 1.04$ & $14.42 \pm 0.71$ & $1.15 \pm 0.68$ & Gxcellent \\
F6 & $31 \pm 0.34$ & $13.91 \pm 0.59$ & $1.11 \pm 0.29$ & Good \\
F7 & $29.8 \pm 0.76$ & $9.45 \pm 0.55$ & $1.18 \pm 0.19$ & Excellent \\
F8 & $32 \pm 0.09$ & $14.41 \pm 0.35$ & $1.15 \pm 0.37$ & Good \\
F9 & $31.5 \pm 0.54$ & $15.71 \pm 0.34$ & & Good \\
\hline
\end{tabular}

${ }^{*}$ Results are expressed as mean $\pm \mathrm{SD}, \mathrm{n}=3$.

\section{Solubility study}

The results of the solubility study are illustrated in table 3 . The data clearly show a significant improvement in the solubility of cefixime after its formulation as a liquisolid powder from sparingly soluble to freely soluble drug, which might be attributed to the increment in surface area and wettability of drug in liquisolid formula F3 in comparison to cefixime powder [26].

Table 3: Solubility data for the cefixime and optimum formula (F3) expressed in $\mathrm{g} / \mathrm{l}$

\begin{tabular}{lll}
\hline & Cefixime powder & F3 \\
\hline pH 7.2 phosphate buffer & $0.029 \pm 0.93$ & $0.375 \pm 0.29$ \\
Distilled water & $0.027 \pm 0.77$ & $0.361 \pm 0.33$ \\
\hline
\end{tabular}

${ }^{*}$ Results are expressed as mean $\pm \mathrm{SD}, \mathrm{n}=3$.

\section{Scanning electron microscope (SEM) study}

Fig. 1 clearly assures the conversion of cefixime trihydrate by liquisolid technique from a crystalline form (A) to the amorphous state (B), which proved the complete solubilization or dispersion of the drug in the liquisolid system that enhances the dissolution of the cefixime from the liquisolid formulas [27].

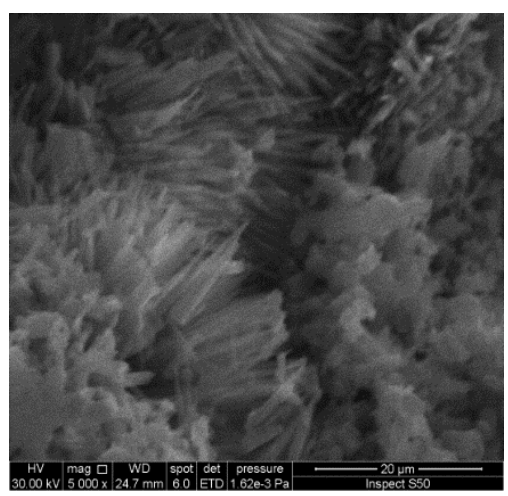

$-\mathbf{A}-$

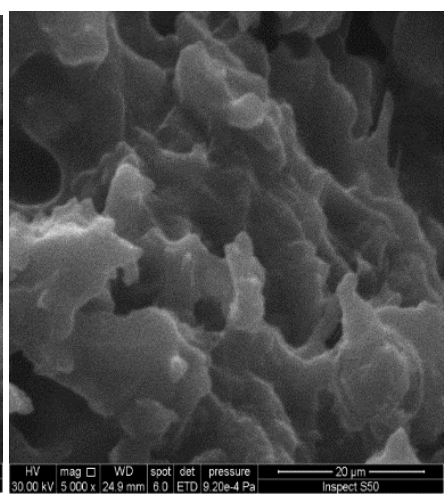

-B-

Fig. 1: SEM image for (A) cefixime trihydrate powder (B) optimum liquisolid formula (F3) containing cefixime trihydrate

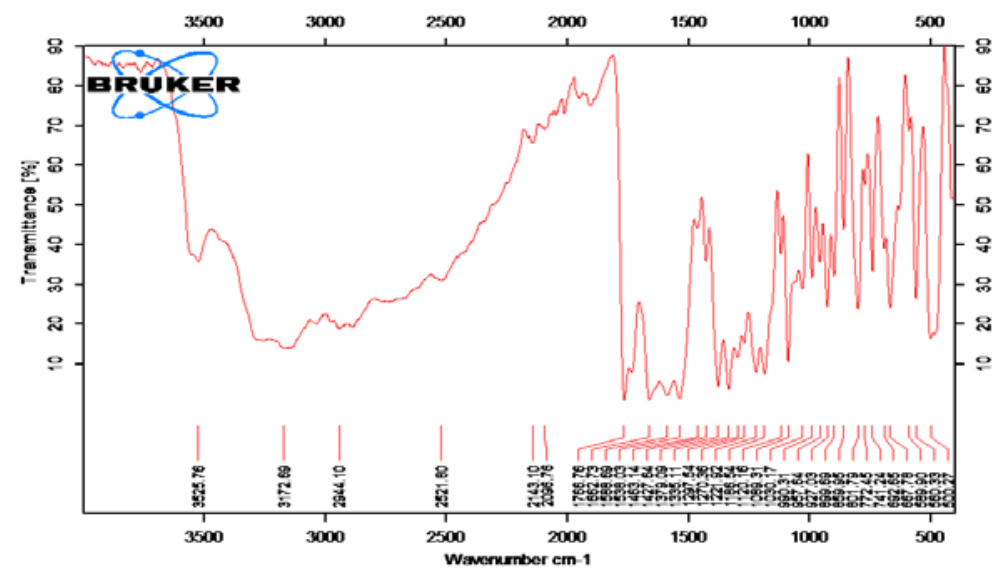

Fig. 2: FT-IR spectrum of cefixime trihydrate 


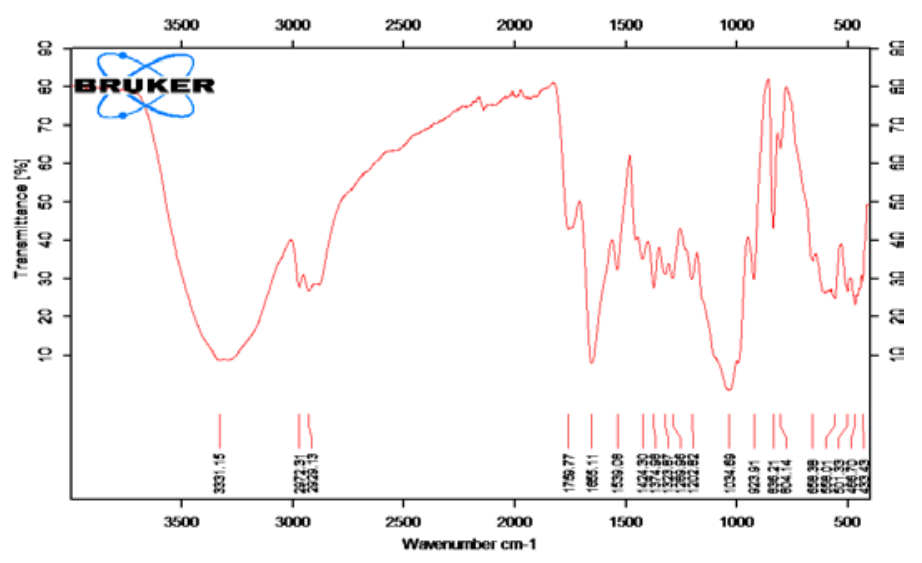

Fig. 3: FT-IR spectrum of optimum liquisolid formula (F3)

\section{Fourier transform infra-red spectroscopy (FT-IR) study}

FT-IR spectrum of cefixime trihydrate and optimum formula (F3) were shown in fig. 2 and 3 respectively. Fig. 1 clearly demonstrates the characteristic peaks of the drug at $3525 \mathrm{~cm}^{-1}$ which results from OH stretching vibration, peaks at 3172 and $2944 \mathrm{~cm}^{-1}$ resulting from $\mathrm{NH}$ stretching vibration of $1^{\circ}$ and $2^{\circ}$ amine, while the stretching vibration of $\mathrm{C}=0$ of ketone, carboxyl and lactam was clearly demonstrated at the bands from $1870-1540 \mathrm{~cm}^{-1}$. On the other hand, a little shifting was observed in the peaks the optimum liquisolid formula (F3) to a lower frequency might be explained by the H-bond formation which might be responsible for solubility enhancement of cefixime in the liquisolid system $[28,29]$.

\section{Weight variations and content uniformity data}

Table 4 demonstrated the weight variation and content uniformity of cefixime liquisolid preparation, the result clearly shows that all formulas complied with the USP specifications, which suggests a uniform distribution of the active ingredients and formulas within the capsule dosage form.

Table 4: Weight variations and content uniformity parameters for cefixime liquisolid capsules (mg)

\begin{tabular}{lll}
\hline Formulas & Weight variation & Content uniformity \\
\hline F1 & $595.4 \pm 0.86$ & $195.2 \pm 0.54$ \\
F2 & $645.8 \pm 0.56$ & $196.5 \pm 0.94$ \\
F3 & $698.04 \pm 0.16$ & $197.6 \pm 0.92$ \\
F4 & $556.32 \pm 0.65$ & $196.53 \pm 0.65$ \\
F5 & $603.4 \pm 0.72$ & $197.4 \pm 0.45$ \\
F6 & $651.63 \pm 0.43$ & $195.8 \pm 0.83$ \\
F7 & $522.3 \pm 0.54$ & $197.04 \pm 0.17$ \\
F8 & $568.34 \pm 0.23$ & $198.34 \pm 0.84$ \\
F9 & $609.52 \pm 0.95$ & $197.05 \pm 0.67$ \\
\hline
\end{tabular}

${ }^{*}$ Results are expressed as mean $\pm \mathrm{SD}, \mathrm{n}=3$.

\section{In vitro disintegration and dissolution test}

Results of In vitro disintegration and dissolution, in addition to the dissolution profiles for cefixime liquisolid formulas are illustrated in table 5 and fig. (4-6). The results clearly show that disintegration time for all formulas was less than 30 min with no significant differences between formulas.

While, the dissolution results demonstrated a significant increment in $\% \mathrm{D}_{45}$ min upon increasing carrier/coat ratio (R) from 6 to 10 in the formulas (F1-F3), (F4-F6) and (F7-F9), which might be attributed to enhanced wicking, deaggregation and disintegration properties that resulted from low liquid/powder ratio, high amount of cellulose and low amount of silica (aerosil) which accompanies higher R values. On the other hand, the data revealed a significant reduction in $\% \mathrm{D}_{45 \mathrm{~min}}$ upon increasing \% drug from $70 \%$ to $80 \%$ in formulas (F1-F3), (F4F6) and (F7-F9) due to the possible precipitation of the drug on aerosil pores at higher concentration, or might be due to the reduced amount of solubilized drug resulting from the lesser amount of solvent used in higher drug concentration.

Therefore, F3 with higher $\mathrm{R}$ value and lower \% of the drug was selected as the optimum formula having $\% \mathrm{D}_{45} \min$ equals to $99.5 \% \pm 0.53$.

Furthermore, the comparative release profile of the optimum liquisolid formula (F3) and the marketed cefixime capsule (200 mg) is illustrated in fig. 7. The fig. shows no significant differences in $\%$ $\mathrm{D}_{45 \mathrm{~min}}$, although, a significant differences were observed in the first 20 min with a greater release rate for F3, which can be explained according to the "Noyes-Whitney" equation and the "diffusion layer model" dissolution theories where the dissolution rate of a drug (DR) is equal to.

$$
\mathrm{DR}=(\mathrm{D} / \mathrm{h}) \mathrm{S}\left(\mathrm{C}_{\mathrm{s}}-\mathrm{C}\right)
$$

Since both D (diffusion coefficient of the drug molecules transported through it) and h (thickness of the stagnant diffusion layer formed by the dissolving liquid around the drug particles) were constants, then DR directly proportional to the $S$ (surface area of the drug available for dissolution) and $\mathrm{C}_{s}-\mathrm{C}$ (concentration gradient where $\mathrm{C}$ is the drug concentration in the bulk of the dissolving medium, and finally $\mathrm{Cs}$ is the saturation solubility of the drug in the dissolution medium).

For this reason, dissolution rate increased in liquisolid systems as it contains a solution of the drug in a suitable solvent (PG), therefore, drug surface available for dissolution was tremendously increased compared to the marketed drug. Another explanation is to the possible increment in the Cs in the case of liquisolid system due to the presence of small quantities of PG that act as cosolvent with the aqueous dissolution medium of the stagnant diffusion layer, resulting in a larger drug concentration gradient (Cs _ C), thereby increasing the dissolution rate [30]. 
Table 5: In vitro disintegration and dissolution parameters for cefixime liquisolid capsules

\begin{tabular}{lll}
\hline Formulas & DT (min) & \% D 45 min (\%) \\
\hline F1 & $27 \pm 0.35$ & $92.07 \pm 0.23$ \\
F2 & $26 \pm 0.41$ & $97.9 \pm 0.41$ \\
F3 & $25 \pm 0.76$ & $99.5 \pm 0.53$ \\
F4 & $26 \pm 0.24$ & $79.6 \pm 0.31$ \\
F5 & $27 \pm 0.34$ & $91.2 \pm 0.18$ \\
F6 & $26 \pm 0.21$ & $95.1 \pm 0.17$ \\
F7 & $25 \pm 0.09$ & $78.5 \pm 0.31$ \\
F8 & $26 \pm 0.45$ & $85.1 \pm 0.24$ \\
F9 & $27 \pm 0.32$ & $85.44 \pm 0.45$ \\
\hline
\end{tabular}

*Abbreviations: Disintegration time (DT), \% drug release after $45 \mathrm{~min}\left(\% \mathrm{D}_{45} \mathrm{~min}\right.$ ). Results are expressed as mean \pm SD, $\mathrm{n}=3$.

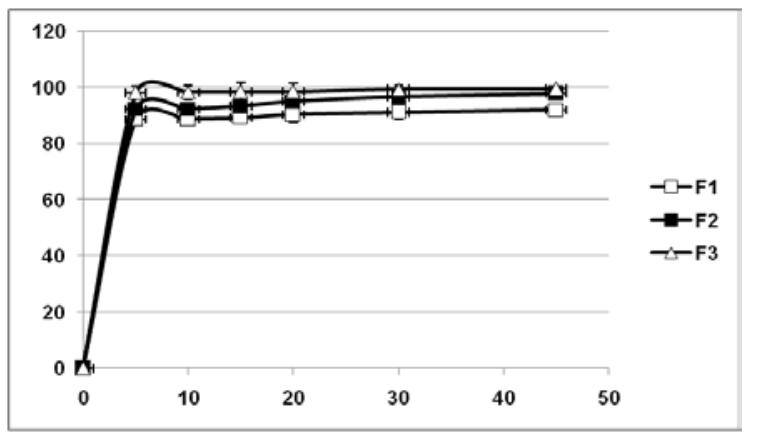

Fig. 4: In vitro release profile for liquisolid formulas (F1-F3) containing $70 \%$ cefixime and with $R$-values $(6,8$ and 10$)$ respectively in phosphate buffer pH 7.2 (Results are expressed as mean $\pm S D, n=3$ )

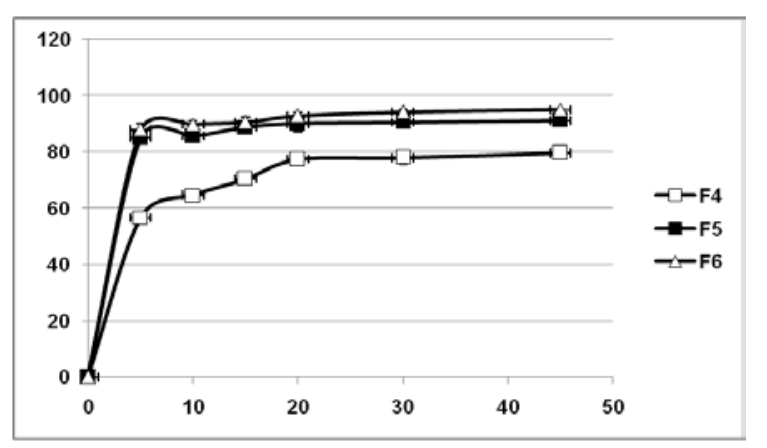

Fig. 5: In vitro release profile for liquisolid formulas (F4-F6) containing $75 \%$ cefixime and with $R$-values $(6,8$ and 10$)$ respectively in phosphate buffer pH 7.2 (Results are expressed as mean $\pm S D, n=3$ )

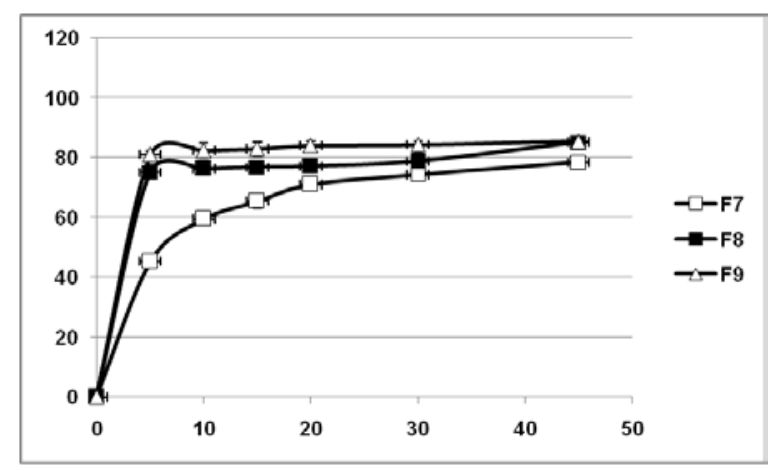

Fig. 6: In vitro release profile for liquisolid formulas (F7-F9) containing $80 \%$ cefixime and with R-values $(6,8$ and 10) respectively in phosphate buffer pH 7.2 (Results are expressed as mean $\pm S D, n=3$ )

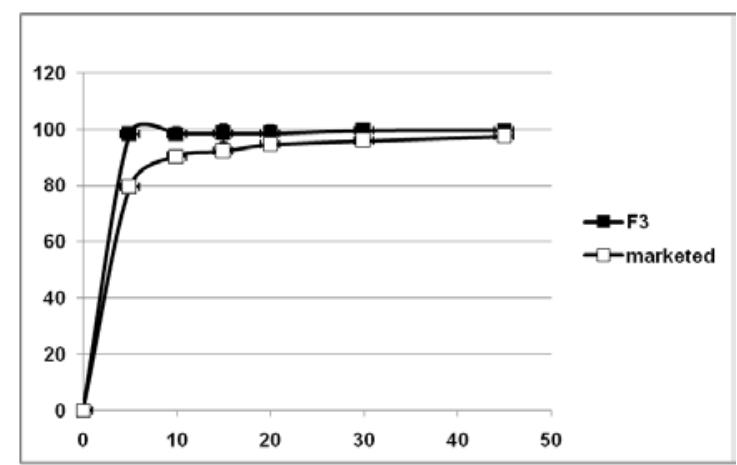

Fig. 7: In vitro release profile for optimum formula (F3) and cefixime marketed drug in phosphate buffer pH 7.2 (Results are expressed as mean $\pm S D, n=3$ )

\section{CONCLUSION}

In this research, it is proved that liquisolid technology can be implemented to enhance the solubility and dissolution of cefixime trihydrate, which might significantly improve its absorption via the oral route leading to increase its bioavailability and might reduce its dose intake.

\section{ACKNOWLEDGMENT}

The authors would like to thank Al-Mustansiriyah University/college of pharmacy/Baghdad/Iraq (www. uomustansiriyh. edu. iq) for presenting this work.

\section{AUTHORS CONTRIBUTIONS}

All the author have contributed equally

\section{CONFLICT OF INTERESTS}

Declared none

\section{REFERENCES}

1. Soujanya BS, Srujan MK, Subrahmanyam KV, Shubhrajit M. Enhancement of solubility of efavirenz by liquisolid compact technique. Int J Innov Pharm Sci Res 2013;1:347-59.

2. Rajkumar G, Kalla M, Swathi RT, Anilkumar V, Indarapu RP. Enhancement of solubility and dissolution rate of ezetimibe through liquisolid technique. Int J Pharm Sci Res 2013;4:3229-38.

3. Mokale V, Naik J, Patil K, Chaudhari R, Khairnar G. Enhancement of solubility with the formulation and in vitro evaluation of oral nateglinide compacts by liquisolid technique. Adv Diabetes Metab 2013;1:57-64.

4. Pavan RK, Karimunnisa SS, Pravin DC. Application of liquisolid technology for enhancing solubility and dissolution of rosuvastatin. Adv Pharm Bull 2014;4:197-204.

5. Shailendra SS, Pritesh P, Sachin I, Praveen S, Biresh S. Formulation and evaluation of curcumin liquisolid tablets. Curr Res Pharm Sci 2012;4:210-4.

6. Ngiik T, Amal AE. Effects of liquisolid formulations on the dissolution of naproxen. Eur J Pharm Biopharm 2009;73:373-84. 
7. Indrajeet DG, Amrit BK, Avinash $\mathrm{HH}$. Evaluation of in vitro dissolution profile comparison methods of sustained release tramadol hydrochloride liquisolid compact formulations with marketed sustained-release tablets. Dig J Nanomater Biostruct 2009;4:651-61.

8. Spireas S, Bolton M. Liquisolid systems and methods of preparing same. United States Patent Application Publication 5,968,550A; 1999

9. Sai SR, Shamili K, Sathesh PR, Subrahmanyam VS. Formulation and in vitro evaluation of efavirenz liquisolid compacts. Int J Pharm Pharm Sci 2016;8:137-43.

10. Suryakant S, Chinam NP, Bhanoji ME. Pharmaceutical drug delivery systems and vehicles. In: Chinam NP, Suryakanta S, Kahnu CP, Muddana EB. editors. An overview of liquisolid technology. India: Woodhead Publishing; 2016. p. 146-60.

11. Satyajit P, Varaprasad R, Priyanka K, Ranjit P. Liquisolid technique: a novel approach for dosage form design. Int J Appl Pharm 2017;9:8-14.

12. Maraie NK, Emad A. Comparative study between liquisolid compacts, liquisolid microsystem and solid dispersion technology for the preparation of immediate release nicardipine tablets. Int J Pharm Sci Rev Res 2017;43:116-26.

13. Sweetman SC. editor. Martindale the complete drug reference. China: Pharmaceutical Press publishing; 2009.

14. Anthony CM, David MO, Brian W. Clarke's analysis of drugs and poisons. London: Pharmaceutical Press; 2005.

15. Patel N. Liquisolid dosage system: a novel approach for dosage formulation. J Pharm Sci Bio-Sci Res 2014;4:125-30.

16. Shrikant M. Liquisolid technique for dissolution and bioavailability enhancement of poorly soluble drugs. Univers J Pharm Res 2017;2:46-53.

17. Spireas S. Liquisolid systems and methods of preparing same, United States Patent Application Publication 5,800,834; 1998.

18. Gilbert SB, Neil RA. The theory and practice of industrial pharmacy. In: Lachman L, Lieberman HA. editors. Tablets. India: CBS Publishing; 2009. p. 293-4.
19. Reecha M, Rajni B, Tejeswini V, Ritima S, Shivali G. Formulation and characterization of matrix tablets using mucilage of tinosporacordifolia as a natural binder. Int J Pharm Pharm Sci 2018;10:22-7.

20. Mogal P, Derle D. Optimization and formulation development of cefixime complex using a spray drying technique: doe approach. J Appl Pharm 2017;9:1-8.

21. Pawar JD, Jagtap RS, Doijad RC, Desai JR, Pol SV, Jadhav VV, et al. Dissolution enhancement of Itraconazole by liquisolid compacts techniques and its in vitro evaluation. Asian J Sci Technol 2017;8:5894-9.

22. Rajesh A, Alpesh Y, Isha S, Sangeeta A, Shailendra B. The liquisolid technique: a review. Int J Pharm Res Bio-Sci 2013;2:464-73

23. Silverstein RM, Webster FX, Kiemle D. editors. Infrared spectrometry. New York: Wiley publishing; 2005.

24. Allen LV, Popovich NG, Ansel HC. editors. Capsule. Philadilphia: Lippincott Williams and Wilkins Publishing; 2011.

25. Mogal P, Deeliprao D. Cefixime, in general, class 4 drug but individually class 2 drug. J Med Physiol Ther 2017;1:1-10.

26. Remeth JD, Kailas KM, Vishwajeet SG, Vijay DH, Vishal RM. Formulation and evaluation of carbamazepine liquisolid compacts using novel carriers. Indian J Pharm Edu 2017;51:69-78.

27. Rania HF, Mohammed AK. Enhancement of famotidine dissolution rate through liquisolid tablets formulation: in vitro and in vivo evaluation. Eur J Pharm Biopharm 2008;69:993-1003.

28. Janakidevi S, Ramanamurthy KV. Formulation and evaluation of cefixime trihydrate matrix tablets using hpmc, sodium $\mathrm{cmc}$, ethyl cellulose. Indian J Pharm Sci 2015;77:321-7.

29. Evangelos K, Georgios K, Aristotelis X, Emmanouel G. Effect of hydrogen bonding interactions on the release mechanisim of felodipine from nanodispersions with polyvinylpyrrolidone. Eur J Pharm Biopharm 2006;63:1-12.

30. Remeth JD, Shashi R, Kailas KM, Vishwajeet SG, Vijay DH. Liquisolid compacts of meloxicam: in vitro and in vivo evaluation. Egypt Pharm J 2017;16:112-20. 